\title{
Boundary effects on localized structures in spatially extended systems
}

\author{
A. Yadav * and D. A. Browne \\ Department of Physics and Astronomy, Louisiana State University, Baton Rouge, LA 70803-4001
}

\begin{abstract}
We present a general method of analyzing the influence of finite size and boundary effects on the dynamics of localized solutions of non-linear spatially extended systems. The dynamics of localized structures in infinite systems involve solvability conditions that require projection onto a Goldstone mode. Our method works by extending the solvability conditions to finite sized systems, by incorporating the finite sized modifications of the Goldstone mode and associated nonzero eigenvalue. We apply this method to the special case of non-equilibrium domain walls under the influence of Dirichlet boundary conditions in a parametrically forced complex Ginzburg Landau equation, where we examine exotic nonuniform domain wall motion due to the influence of boundary conditions.
\end{abstract}

PACS numbers: 82.40.-g,05.70.Ln

\section{INTRODUCTION}

Models of non-linear spatially extended systems exhibit a variety of spatial and temporal pattern forming phenomena. A subclass of these patterns are spatially localized structures 1] that include pulses, solitons, fronts, and domain walls. The standard analysis of these localized structures assumes that, on large length and time scales, they can be treated as "coherent objects" [1], with effective parameters like position, and velocity attributed to them. A perturbative expansion about this isolated coherent object profile is then used to understand its response to external forces, interaction with other localized structures 2, 3], noise, or internal instabilities [4, 5]. Perturbative calculations reduce the original nonlinear problem to a series of linear problems that require consistency criteria known as solvability conditions for their solution. Typically, the solution of a linear equation $L \phi=\psi$, requires the orthogonality of $\psi$ to the zero modes $\chi$, ie., $(\psi, \chi)=0$, in the null space of the adjoint homogeneous problem $L^{\dagger} \chi=0$.

Often, the symmetries in a particular system are responsible for the zero modes of the operators obtained after a perturbative expansion. For instance, since a localized structure profile and the same profile translated infinitesimally are both solutions of the underlying nonlinear equation, the difference of the two profiles provides a zero (neutral or Goldstone) mode. Strictly, the zero mode is the derivative of the localized structure profile, and the underlying symmetry is translation invariance. Zero modes extracted from symmetry arguments may then be employed straightforwardly into solvability integrals.

The argument above, based on translational invariance, works if the system size is infinite. For a localized structure near a system boundary, due to the relevant boundary conditions that have to be imposed there, the localized structure solution and its infinitesi-

*Electronic address: yadav@phys.lsu.edu mally translated counterpart are no longer solutions of the same equation. Hence, translational invariance is broken. Therefore, in this case, one has to contend, not only with the incorporation of the boundary data into the solvability condition, but also the appropriate treatment of broken translation invariance.

Most treatments of localized structures follow analytical techniques that fall in the realm of moving boundary approximations [6]. A common feature to these approximations, for instance, in excitable waves [7], or bistable fronts [8, 9, 10], is the separation of the description of the localized structure into an "inner region" and "outer region". The inner region, characterized by short spatial scales and fast time scales, captures the internal dynamics of the localized structure. In contrast, the dynamics of the localized structure as a whole is captured by the long spatial and time scales comprising the outer problem. The solvability integrals in moving boundary type approximations occur in the inner problem. Since it is the fields in the outer region that mediate the interaction with the boundary [11, 12], the boundary data is not incorporated into solvability conditions arising in the inner problem. There are ample situations however, where it may not be possible to have separate inner and outer regions of a localized structure by manipulating relevant system parameters [14]. In such cases, the boundary data must be directly incorporated into the solvability condition.

In this paper, through an appropriately chosen adjoint operator $L^{\dagger}$ defined for the semi-infinite system (localized structure near a boundary), we develop techniques that not only include the boundary data into the solvability condition, but also directly incorporate the effects of broken translational invariance into it. We accomplish this by extending the definition of the Goldstone mode to include the possibility that the corresponding eigenvalue be non-zero, with its magnitude dependent on how close the localized structure is to the boundary. This leads further to a modified solvability criteria.

As a case study, we develop our techniques in the context of reaction-diffusion systems and apply it to non-equilibrium domain walls (fronts) found in bistable regimes. In bistable reaction-diffusion systems, fronts 
connecting the two homogeneous steady states can undergo a bifurcation, called a front bifurcation, where a stationary Ising front loses stability to two counterpropagating Bloch fronts 14]. This bifurcation can be regarded as an internal instability of the Ising front, the localized structure about which a perturbative expansion is carried out to obtain the propagating Bloch wall solution. This bifurcation, also known as the Ising-Bloch bifurcation, has been observed in several systems, like chemical reactions [4, 15, 16] and also in liquid crystals [17, 18].

In a recent work [12], we examined the influence of boundaries on Ising-Bloch fronts in a FitzHugh-Nagumo (FHN) reaction diffusion model. We were able to derive order parameter equations (OPE) for front dynamics, where the fronts were perturbed by the imposition of Dirichlet and possibly other boundary conditions at the boundaries. This derivation for the two component FHN model required restrictive assumptions about the relative size of the fronts for the two concentration fields, allowing for the use of moving boundary approximation like singular perturbation methods detailed in 8, 9]. These singular perturbation techniques are quite versatile, predicting exotic phenomena like front reversal, trapping, and oscillation at the boundary. However, as observed earlier, we wish to examine the effects of boundary data on localized structures, where moving boundary type approximations are not applicable, and the explicit incorporation of boundary data in a solvability condition is required.

In the next section we discuss the extension of the solvability condition to incorporate boundary data and broken translational invariance via the extension of the Goldstone mode in a generic system exhibiting a localized structure. In Sec. III, we describe the modification of the slow manifold of a generic Ising-Bloch front due to boundary effects. In Sec. IV, we apply our method of solvability condition extension to study the effects of finite size and Dirichlet boundary conditions on the dynamics of Ising-Bloch fronts in a parametrically forced complex Ginzburg Landau equation (CGLE) 5, 14]. An important reason behind this choice is its experimental context, modeling Ising-Bloch fronts in Liquid crystals subjected to rotating magnetic fields [17, 18]. Liquid crystal systems are ideal candidates to study boundary effects, as lateral boundary conditions may be imposed in a controlled manner by appropriate electric fields $[19$. Another experimental test bed is presented Ref. [20], in the form of coupled non-linear electrical oscillators, where the application of boundary conditions requires a minor and straightforward variation of the original circuit. In Sec. $\mathrm{V}$ we discuss in detail the implications of the derived order parameter equations for the parametrically forced CGLE. In Sec. VI we present our conclusions.

\section{GOLDSTONE MODES AND SOLVABILITY CRITERIA}

Consider a general non-linear PDE,

$$
\partial_{t} U=\mathcal{L} U+N(U)
$$

where $U(x, t)$ is the solution vector, $\mathcal{L}$ are the linear terms, and $N(U)$ are the non-linear terms. Let $U_{0}(x)$ be a stationary localized solution of Eq. (1), with the asymptotic behavior $U(x) \rightarrow 0 ; x \rightarrow \pm \infty$. In principle, $U_{0}(x)$ also encompasses uniformly translating localized structures, which are stationary in a co-moving frame.

Due to translational invariance in the system, one has $A(x)=U_{0 x}$, the derivative with respect to $x$ of the localized structure profile, as the zero eigenvalue (neutral or Goldstone) mode of the operator $£=\mathcal{L}+N^{\prime}\left(U_{0}\right)$. Also, it is reasonable to expect that due to translational invariance $£^{\dagger}$ has a corresponding zero eigenvector, given by the solution of $£^{\dagger} A^{\dagger}=0$. A detailed discussion of this issue may be found in [13] and the references therein.

While examining the stability of $A=U_{0 x}$ to perturbations, which may include a small external perturbation $p(U, x)$ added onto Eq. (1), one obtains,

$$
\begin{aligned}
& {\left[\mathcal{L}+N^{\prime}\left(U_{0}\right)\right] \delta U=f } \\
f= & \partial_{t}(\delta U)-\left\{N^{\prime \prime}\left(U_{0}\right)(\delta U)^{2} / 2+p\left(U_{0}, x\right)\right. \\
+ & \left.p^{\prime}\left(U_{0}, x\right) \delta U+p^{\prime \prime}\left(U_{0}, x\right)(\delta U)^{2} / 2+\mathcal{O}\left[(\delta U)^{3}\right]\right\}
\end{aligned}
$$

where $\delta U$ is the small deviation from the localized structure profile. Realizing that the operator $£=\mathcal{L}+N^{\prime}\left(U_{0}\right)$ has a Goldstone mode, the solvability of Eq. (2) requires,

$$
\left(f, A^{\dagger}\right)=0 .
$$

The brackets indicate an inner product or the projection of the dynamical terms $f$ onto the Goldstone mode (its corresponding adjoint) $A^{\dagger}$. Equation. (3) represents the generic response of a localized structure to a wide variety of perturbations, both internal and external. From an informal and intuitively appealing point of view, the Goldstone mode with its associated zero eigenvalue is a slow (relevant) mode, which coupled with other slow modes in the system, should dominate the dynamics. The projection in Eq. (3) is a formal prescription to capture this slow dynamics.

Let a localized structure be located near a boundary at $x=-l$, with the origin fixed at the position of the localized structure. Although, $A^{\dagger}$ is still a solution of $£^{\dagger} A^{\dagger}=0$ in this case, it does not assume the homogeneous boundary value $A^{\dagger}(-l)=0$. Consequently, $A^{\dagger}$ is no longer the zero eigenvector of the adjoint homogeneous problem in the semi-finite interval $[-l, \infty]$. However, we still expect $A^{\dagger}$ to play a central role in the dynamics of the localized structure, all be it in a slightly modified form $A_{l}^{\dagger}=A^{\dagger}+\delta A_{l}^{\dagger}$. The subscript $l$ denotes the proximity of the localized structure to the boundary, and $\delta A_{l}^{\dagger}$ is a proximity dependent correction to $A^{\dagger}$. We require that in the limit $l \rightarrow \infty, A_{l}^{\dagger} \rightarrow A^{\dagger}$, and $\delta A_{l}^{\dagger} \rightarrow 0$. 
This requirement is reasonable on physical grounds. The slow dynamics of the localized structure far away from the boundary involves $A^{\dagger}$ as a relevant constituent by virtue of it being a slow mode. As the localized structure gradually nears the boundary, we still expect $A^{\dagger}$, in its modified form $A_{l}^{\dagger}$, to be the relevant (slow) constituent of the dynamics.

$A_{l}^{\dagger}$ may be determined in two possible ways. Firstly, we may extract $A_{l}^{\dagger}$ as the solution of

$$
£^{\dagger} A_{l}^{\dagger}=0, \quad A_{l}^{\dagger}(-l)=0, \quad A_{l}^{\dagger}(\infty)=0,
$$

with the implication that $A_{l}^{\dagger}=A^{\dagger}+\delta A_{l}^{\dagger}$ is still a zero eigenvector in the finite system. Or we may extract $A_{l}^{\dagger}$ as a solution of

$$
£^{\dagger} A_{l}^{\dagger}=\lambda_{l} A_{l}^{\dagger}, \quad A_{l}^{\dagger}(-l)=0 . \quad A_{l}^{\dagger}(\infty)=0 .
$$

Thus, as the localized structure gradually closes in on a boundary, the zero eigenvector $A^{\dagger}$ is modified to $A_{l}^{\dagger}$, and the zero eigenvalue gradually migrates away from zero, assuming the value $\lambda_{l}$. Hence, as $l \rightarrow \infty, \lambda_{l} \rightarrow 0$, and $A_{l}^{\dagger} \rightarrow A$.

The first scenario is easily discarded using uniqueness arguments. If Eq. (4) is obeyed, then $\delta A_{l}^{\dagger}$ should obey, $£^{\dagger} \delta A_{l}^{\dagger}=0, \delta A_{l}^{\dagger}(-l)=-A^{\dagger}(-l), \delta A_{l}^{\dagger}(\infty)=0$, with the unique solution $\delta A_{l}^{\dagger}=-A^{\dagger}$. Therefore, since $A_{l}^{\dagger}=A^{\dagger}+\delta A_{l}^{\dagger}$, Eq. (4) only has the trivial solution $A_{l}^{\dagger}=0$ (the uniqueness of homogeneous and inhomogeneous problems involving linear differential operators on semi-infinite intervals can be proved by a transformation that takes the semi-infinite interval into a finite interval, followed by the utilization of theorems on uniqueness available for finite intervals. We provide a proof in Appendix A for the CGLE that is studied in detail in later sections. Moreover, such a transformation may also be applied to operators with an asymptotic structure similar to that of the CGLE). This leads us to conclude that the modification of $A^{\dagger}$ in a finite system is appropriately represented by Eq. (5).

For arbitrary functions $u$ (not the field $U$ in Eq. (10) and $v$, and using integration by parts, we have,

$$
\begin{aligned}
(£ u, v) & =\left(u, £^{\dagger} v\right)+v(b) u_{x}(b)-v(a) u_{x}(a) \\
& +v_{x}(a) u(a)-v_{x}(b) u(b),
\end{aligned}
$$

where we assume for simplicity that $£$ is a reactiondiffusion type operator comprised of second order differential terms only. $x=a$ and $x=b$ are arbitrary boundary points. If needed, one may evaluate surface terms for more general operators using integration by parts.

For the localized structure $a=-l$ and $b=\infty$. We invoke Eq. (5) and substitute $v=A_{l}^{\dagger}, u=\delta U_{l}$ (the subscript $l$ denotes that $\delta U$ is now considered in a finite system) in Eq. (6), to obtain,

$$
\begin{aligned}
\left(£ \delta U_{l}, A_{l}^{\dagger}\right) & =\left(f, A_{l}^{\dagger}\right)=\left(\delta U_{l}, \lambda_{l} A_{l}^{\dagger}\right)+A_{l x}^{\dagger}(-l) \delta U_{l}(-l) \\
& -A_{l x}^{\dagger}(\infty) \delta U_{l}(\infty) .
\end{aligned}
$$

This is the sought after finite system extension of the solvability criteria Eq. (3). Also, as $l \rightarrow \infty$, Eq. (7) reduces to $\left(f, A^{\dagger}\right)=0$. Since $£$ is obtained by linearizing about the localized structure $U_{0}(x), \delta U_{l}(-l)$ is simply the difference $U(-l)-U_{0}(-l)$, where $U(-l)$ is the Dirichlet boundary value imposed on field $U$, the solution of Eq. (1).

The extension Eq. (7), tailored to incorporate nonhomogeneous Dirichlet boundary conditions on the field $U$, is not unique. For instance, one may consider the effects of non-homogeneous Neumann boundary conditions on the field $U$ by requiring that $A_{l}^{\dagger}$ obeys

$$
£^{\dagger} A_{l}^{\dagger}=\lambda_{l} A_{l}^{\dagger}, \quad A_{l x}^{\dagger}(-l)=0, \quad A_{l x}^{\dagger}(\infty)=0 .
$$

Here, the derivatives, rather than $A_{l}^{\dagger}$ itself, assume zero values at the boundary. Furthermore, an extension $A_{l}^{\dagger}$ for a general set of homogeneous boundary conditions, with homogeneous Dirichlet and Neumann boundary conditions as special cases, may also be developed. Next, we apply the techniques and criteria developed so far to analyze non-equilibrium Ising-Bloch fronts, as the fronts interact with the system boundary.

\section{BOUNDARY EFFECTS IN A GENERIC ISING-BLOCH SYSTEM}

Ising-Bloch fronts provide an interesting arena to apply the methods developed in the last section. Along with the usual Goldstone mode associated with translational invariance, the slow manifold for Ising-Bloch fronts also includes a spatially localized slow mode responsible for the Ising-Bloch bifurcation 5, 21, 22. . Chirality preserving stationary Ising fronts 14], bifurcate into a pair of chirality broken, counter-propagating Bloch fronts. The slow manifold for Ising-Bloch fronts comprised of the Goldstone and chirality breaking modes, manifests itself in the form of order parameter equations (OPE) [4, 5, 22. for the order parameters, front velocity and front position. The front velocity is a measure of the effects of the chirality breaking mode. The Goldstone mode captures front translations by infinitesimal changes in the front position, the other order parameter. We seek the coupling between these order parameters induced by the boundary data and broken translational invariance.

A generic Ising front denoted by $U_{0}(x)$, gives the Goldstone mode $U_{0 x}$. Close to the Ising-Bloch bifurcation threshold, propagating Bloch wall solutions are regarded as perturbations of the stationary Ising wall solution [14]. The front velocity $c$ controls the strength of these perturbations. Therefore, expanding the deviation $\delta U$ in powers of $c$, we have,

$$
\begin{aligned}
U_{b} & =U_{0}+\delta U \\
& =U_{0}+c \delta U_{1}+c^{2} \delta U_{2}+c^{3} \delta U_{3}+. .,
\end{aligned}
$$

with the perturbed Bloch wall solution $U_{b}$. 
For convenience we transform into a frame of reference moving along with the Bloch wall. This transformation amounts to $\partial_{t}(\delta U) \rightarrow \partial_{t}(\delta U)-c\left(U_{0 x}+\delta U_{x}\right)$. Invoking Eq. (2) and substituting into it the expansion of $\delta U$, while at the same time disregarding the influence of any external perturbation $p(U, x)$, we obtain,

$$
\begin{gathered}
£\left[c \delta U_{1}+c^{2} \delta U_{2}+c^{3} \delta U_{3}\right]=\partial_{t}\left(c \delta U_{1}\right) \\
-c\left[U_{0 x}+c \delta U_{1 x}+c^{2} \delta U_{2 x}\right]-c^{2} N_{2}-c^{3} N_{3}+\cdot \cdot
\end{gathered}
$$

$N_{2}$ and $N_{3}$ represent the coefficients of second order and third order velocity terms respectively.

Equating terms which are first order in velocity $c$ in Eq. (10), we obtain,

$$
£ \delta U_{1}+U_{0 x}=0 .
$$

This means that $£$ has a double zero eigenvalue at the Ising-Bloch bifurcation threshold [5, 22]. Therefore, along with the zero Goldstone mode, we have another eigenvalue that passes through zero at the bifurcation. The Goldstone mode $U_{0 x}$ and the generalized eigenvector $\delta U_{1}$ obtained from Eq. (11), span the slow manifold. The chirality breaking mode is then constructed as a linear combination of these two modes [5].

Employing the projection criteria Eq. (3) for an IsingBloch front close to the bifurcation threshold, ie., the solvability of Eq. (10), results in,

$$
\begin{aligned}
\left(\delta U_{1}, A^{\dagger}\right) \partial_{t} c & =c\left(U_{0 x}, A^{\dagger}\right)+c^{2}\left(\delta U_{1 x}+N_{2}, A^{\dagger}\right) \\
& +c^{3}\left(\delta U_{2 x}+N_{3}, A^{\dagger}\right)+\cdots
\end{aligned}
$$

This is the generic OPE for the velocity of Ising-Bloch fronts close to the bifurcation threshold. The particular form of the inner products in Eq. (12) is system specific. If one assumes further symmetries in the system, for example $U \rightarrow-U$, inner products that are coefficients of even powers of the velocity in Eq. (12) vanish, resulting in the normal form of a pitchfork bifurcation. The inner product $\left(U_{0 x}, A^{\dagger}\right)$ in Eq. (12) controls the distance from the Ising-Bloch bifurcation threshold, where for consistency (Ising-Bloch bifurcation is a pitchfork) it is further required that $\left(U_{0 x}, A^{\dagger}\right) \sim c^{2}, \partial_{t} c \sim c^{3}[5,22$. Hence, all the terms in Eq. (12) are of size $c^{3}$.

We invoke the extended solvability criteria Eq. (7) to evaluate the effects of boundary data on the dynamics of Ising-Bloch fronts. For generic Ising-Bloch fronts interacting with boundaries where Dirichlet data is present, the extended solvability criteria assumes the form,

$$
\begin{aligned}
\left(\delta U_{1 l}, A_{l}^{\dagger}\right) \partial_{t} c & =c\left(U_{0 x}, A_{l}^{\dagger}\right)+c^{2}\left(\delta U_{1 l x}+N_{2}, A_{l}^{\dagger}\right) \\
& +c^{3}\left(\delta U_{2 l x}+N_{3}, A_{l}^{\dagger}\right) \\
& +\lambda_{l}\left(c \delta U_{1 l}+c^{2} \delta U_{2 l}+c^{3} \delta U_{3 l}+\cdots, A_{l}^{\dagger}\right) \\
& +A_{l x}^{\dagger}(-l) \delta U_{l}(-l)-A_{l x}^{\dagger}(\infty) \delta U_{l}(\infty) .(13)
\end{aligned}
$$

In contrast to earlier works [4, 5, 22] focused on the effects of external perturbations, $p(U, x)$, on the slow manifold, the constituent modes of the slow manifold require appropriate modifications in order to capture the effects arising due to confinement by boundaries. While, the modification of the adjoint Goldstone mode $A^{\dagger}$ to $A_{l}^{\dagger}$ is generic to any confined localized structure, or alternatively, a localized structure being considered in the vicinity of system boundaries, the modification of the generalized eigenvector $\delta U_{1}$ to $\delta U_{1 l}$ is a unique characteristic of Ising-Bloch fronts.

Simplifications to the slow manifold Eq. (13) are made by the following observations. Consider the term, $f_{0}=$ $\lambda_{l}\left(c \delta U_{1 l}+c^{2} \delta U_{2 l}+c^{3} \delta U_{3 l}+\cdots, A_{l}^{\dagger}\right)$, on the right hand side of Eq. (13). The inner product $f_{1}=\lambda_{l}\left(c \delta U_{1 l}, A_{l}^{\dagger}\right)$ has the largest contribution since it involves the first power of the velocity $c$. Now, as mentioned before, all terms should be of size $c^{3}$, a requirement imposed for the Ising-Bloch bifurcation to be a pitchfork. Therefore, $f_{1} \sim \lambda_{l} c \sim c^{3}$, implying $\lambda_{l} \sim c^{2}$. Moreover, the size of $\lambda_{l}$ is controlled by the distance of the Bloch fronts from the boundary. If the front is far away from the boundary, that is, if $\lambda_{l} \sim \mathcal{O}\left(c^{3}\right)$, then $f_{1} \sim \mathcal{O}\left(c^{4}\right)$, and its contribution to Eq. (13) can be neglected. As the front moves towards the boundary, so that $\lambda_{l} \sim c^{2}$, then $f_{1} \sim c^{3}$ contributes to Eq. (13), and the ensuing front dynamics. If the front gets too close to the boundary, ie., $\lambda_{l} \sim c$, then $f_{1} \sim c^{2}$, and the scaling requiring that all the terms be of size $c^{3}$ breaks down. In other words, if $\lambda_{l} \sim c$, the effects of the boundary are too strong for them to be accurately considered as small perturbations on the dynamics of IsingBloch fronts. Consequently, the size of $\lambda_{l}$ serves as a measure of the strength of the boundary perturbation. In light of the present discussion, Eq. (13) simplifies to

$$
\begin{aligned}
\left(\delta U_{1 l}, A_{l}^{\dagger}\right) \partial_{t} c & =c\left(U_{0 x}, A_{l}^{\dagger}\right)+c^{2}\left(\delta U_{1 l x}+N_{2}, A_{l}^{\dagger}\right) \\
& +c^{3}\left(\delta U_{2 l x}+N_{3}, A_{l}^{\dagger}\right) \\
& +\lambda_{l}\left(c \delta U_{1 l}, A_{l}^{\dagger}\right) \\
& +A_{l x}^{\dagger}(-l) \delta U_{l}(-l) .
\end{aligned}
$$

The surface terms at infinity contribute zero, since by construction $A_{l}^{\dagger}(\infty)=0$.

\section{BOUNDARY EFFECTS IN THE PARAMETRICALLY FORCED CGLE}

The CGLE reads,

$$
\partial_{\tau} F=(\gamma+i \nu) F-|F|^{2} F+\mu F^{*}+\partial^{2}{ }_{X} F+\alpha .
$$

Equation. (15) and its generalizations [3, 5, 14] have been thoroughly analyzed in the context of the Ising-Bloch bifurcation. The field $F$ may be regarded as the amplitude of diffusively coupled auto oscillators that oscillate above the Hopf bifurcation threshold determined by the parameter $\gamma . \mu$ represents the strength of parametric forcing at twice the natural frequency, and $\nu$ is the detuning. The parameter $\alpha$, which models forcing at the natural frequency of the system, breaks the $(F \rightarrow-F)$ symmetry. As a result, the pitchfork normal form of the Ising-Bloch 
bifurcation for $\alpha=0$ unfolds into a saddle node for a non-zero $\alpha$.

We briefly recount the results of $[5]$ concerning the dynamics of Ising-Bloch fronts in the parametrically forced CGLE valid for an infinite system. This lays down the framework for the subsequent consideration of finite system sizes and boundary effects.

For $\alpha=0$ and in the bistable regime determined by the constraints, $|\nu|\langle\mu, \gamma\rangle-\sqrt{\mu^{2}-\nu^{2}}$, Eq. (15) possesses a stationary Ising wall solution $F_{I}=$ $\sqrt{\kappa} \tanh (\sqrt{\kappa / 2 X}) e^{i \phi}$. Here $\kappa=\gamma+\sqrt{\mu^{2}-\nu^{2}}$ and $\phi$ is obtained by solving $\sin (2 \phi)=\nu / \mu$. Bloch wall solutions of Eq. (15) are then obtained as a perturbation to the Ising wall,

$$
F_{b}(x, t)=\sqrt{\kappa}[\tanh (x)+u(x, t)+i w(x, t)] e^{i \phi},
$$

where the space-time scaling $t=\kappa \tau / 2, x=\sqrt{\kappa / 2} X$ is introduced by the authors, resulting in,

$$
\begin{aligned}
£=\left[\begin{array}{cc}
D_{1} & -4 \nu / \kappa \\
0 & D_{2}-3+4 \gamma / \kappa
\end{array}\right], \\
D_{1}=\partial_{x}^{2}+2-6 \tanh ^{2}(x), \\
\quad D_{2}=\partial_{x}^{2}+1-2 \tanh ^{2}(x), \\
\tilde{N}=-2 \tanh (x)\left[\begin{array}{c}
3 u^{2}+w^{2} \\
2 u w
\end{array}\right]-2\left[\begin{array}{c}
u^{3}+u w^{2} \\
w^{3}+w u^{2}
\end{array}\right] .
\end{aligned}
$$

For clarity and continuation of the conventions used in the previous sections, we stress the following points. Firstly, we recognize that $\delta U=\{u, w\}^{T}$. Secondly, $\delta U$ obeys

$$
\partial_{t} \delta U=£ \delta U+\tilde{N}
$$

which when compared with Eq. (2), leads to the realization that $\tilde{N}=N^{\prime \prime}\left(U_{0}\right)(\delta U)^{2} / 2+\mathcal{O}\left[(\delta U)^{3}\right.$. Thirdly, $£$ is obtained by linearizing about the solution $U_{0}(x)$. In the present case the stationary solution is the Ising wall $F_{I}(x)=\sqrt{\kappa} \tanh (x) e^{i \phi}$, and $U_{0}(x)=\tanh (x)$, where the constant factor $\sqrt{\kappa} e^{i \phi}$ should be dropped if the perturbation $\delta U=\{u, w\}^{T}$ is defined through Eq. (16).

For the specific case of the parametrically forced CGLE, one has [5],

$$
\begin{aligned}
& \delta U_{1}=\left[\begin{array}{c}
\frac{8}{3 \pi} I_{11}(x)-I_{12}(x) \\
\frac{8 \gamma}{9 \pi \nu} \operatorname{sech}(\mathrm{x})
\end{array}\right], \quad U_{0 x}=\left[\begin{array}{c}
\operatorname{sech}^{2}(x) \\
0
\end{array}\right], \\
& \text { and } \quad A^{\dagger}=\left[\begin{array}{c}
\frac{9\left(\mu_{c}-\mu\right) \mu_{c}}{\pi \gamma \nu} \operatorname{sech}^{2}(x) \\
\operatorname{sech}(\mathrm{x})
\end{array}\right] .
\end{aligned}
$$

Substituting these vectors into Equation. (12) gives [5],

$$
\partial_{t} c=\frac{27\left(\mu_{c}-\mu\right) \mu_{c}}{4 \gamma^{2}} c-\left(\left[\frac{8 \gamma}{9 \pi \nu}\right]^{2}+0.36\right) c^{3}
$$

Eq. (19) possesses three stationary states, two counterpropagating Bloch walls and a stationary Ising wall. These steady states exchange stability via the IsingBloch bifurcation at the critical bifurcation parameter $3 \mu_{c}=\sqrt{9 \nu^{2}+\gamma^{2}}$. The components of the vectors $\delta U=c \delta U_{1}+c^{2} \delta U_{2}+. ., U_{0}$ and $A^{\dagger}$, in an infinite system, exponentially decay to zero as one moves away from the front both to the left and to the right. This signifies that Ising and Bloch walls are localized structures that are not influenced by boundary conditions imposed on either boundary sufficiently far away. Furthermore, no explicit dependence on $x$ in Eq. (19) indicates translational invariance, a residue of infinite system size.

We now calculate $A_{l}^{\dagger}$ and the associated value of $\lambda_{l} . A_{l}^{\dagger}$ satisfies the boundary conditions $A_{l}^{\dagger}(-l)=0, A_{l}^{\dagger}(\infty)=0$ (homogeneous problem), since we wish to examine the influence of Dirichlet boundary conditions on $U$ (nonhomogeneous problem). Close to the bifurcation threshold determined by the magnitude of $\mu_{c}-\mu$, the operator $£^{\dagger}$ has the form

$$
\begin{aligned}
£^{\dagger} & =\left[\begin{array}{cc}
D_{1} & 0 \\
-\nu / \gamma & D_{2}
\end{array}\right]+\frac{27 \mu_{c}\left(\mu-\mu_{c}\right)}{4 \gamma^{2}}\left[\begin{array}{cc}
0 & 0 \\
\nu / \gamma & -1
\end{array}\right] \\
& =£_{1}^{\dagger}+\left(\mu-\mu_{c}\right) £_{2}^{\dagger} .
\end{aligned}
$$

The operator $£_{2}^{\dagger}$ is a perturbative correction to the operator $£_{1}^{\dagger}$, since $\mu-\mu_{c} \sim c^{2}$. Hence, we first examine $£_{1}^{\dagger}$ the dominant term in $£^{\dagger}$.

The operators $D_{1}$ and $D_{2}$ populate the diagonals of $£_{1}^{\dagger}$, and possess zero eigenvectors given by $Z_{1}=\operatorname{sech}^{2}(x)$ and $Z_{2}=\operatorname{sech}(x)$ respectively, in an infinite system. These eigenvectors satisfy the constraint of being zero at positive and negative infinity. Imagine a traveling Bloch front sufficiently distant from the boundary, where Dirichlet boundary conditions are imposed. The front does not sense the boundary and the condition $D_{1} Z_{1}=D_{2} Z_{2}=0$ holds. This is because the solutions $Z_{1}$ and $Z_{2}$ exponentially approach zero on either side of the front. As the front closes in on the boundary, such that it is barely able to sense it $\left(Z_{1}\right.$ and $Z_{2}$ have small finite values at the boundary), the eigenvectors $Z_{1}$ and $Z_{2}$ are modified to $Z_{1 l}$ and $Z_{2 l}$ by constraining them to have zero values at the boundary. Meanwhile, in a semi-infinite or finite domain, the only solutions to $D_{1} Z_{1 l}=D_{2} Z_{2 l}=0$ which have a zero value at both boundaries are the trivial solutions $Z_{1 l}=Z_{2 l}=0$ (uniqueness arguments). Hence, requiring that the solutions $Z_{1 l}\left(Z_{2 l}\right)$ are only slight modifications of $Z_{1}\left(Z_{2}\right)$ and are not trivial zero solutions demands that these solutions obey $D_{1} Z_{1 l}=\lambda_{1 l} Z_{1 l}$ and $D_{2} Z_{2 l}=\lambda_{2 l} Z_{2 l}$.

Figure. 1(a) shows the plot of $Z_{1}$ in grey, where the left boundary is at a finite distance $l$ from the peak. $Z_{1}$ has a 
finite nonzero value at the boundary. We require that the modified eigenvector $Z_{1 l}$ have a zero value at the boundary and not be all that different from $Z_{1}$ elsewhere. We make the ansatz that this can be accomplished by subtracting from $Z_{1}$ its image to the left of the boundary. Therefore, we have, $Z_{1 l}=\operatorname{sech}^{2}(x)-\operatorname{sech}^{2}(x+2 l)$. Figure. 1(b) shows a good agreement between our guess and the actual numerically evaluated $Z_{1 l}$. This is so because in the asymptotic limit $\exp 2 x>>1, D_{1}=\partial_{x}^{2}-4$, and the image is approximately a zero eigenvector of this operator in the same limit.

Introducing images into a semi-infinite problem is by no means a coincidence. Images are a common occurrence whenever boundary data is involved. For the extension $A_{l}^{\dagger}$ (correspondingly $Z_{1 l}$ and $Z_{2 l}$ ) to assume a zero value at the boundary, the introduction of the image becomes a natural necessity. Furthermore, we wish to stress that the concept of images is quite general in its utility. Extensions of Goldstone modes can be readily obtained for other systems, with linear operators having similar properties of exponential decay asymptotics.

An upper bound, $\lambda_{1 l}^{\uparrow}$, on the eigenvalue $\lambda_{1 l}$, is easily obtained by a variational principle, given by,

$$
\left|\lambda_{1 l}\right|<\left|\lambda_{1 l}^{\uparrow}\right|=\left(Z_{1 l}, D_{1} Z_{1 l}\right) /\left(Z_{1 l}, Z_{1 l}\right) .
$$

A more refined variational guess of $Z_{1 l}$ may be made by introducing an extra parameter $a_{1}$. Consequently, we have $Z_{1 l}=\exp \left(a_{1} x\right)\left[\operatorname{sech}^{2}(x)-\operatorname{sech}^{2}(x+2 l)\right]$. Manipulation of this parameter provides a better guess of the change in shape of the peak in the actual modified eigenvector $Z_{1 l}$. Figure. 2(a) compares the numerical and variationally calculated eigenvalues as a function of the distance $l$ of the front from the boundary. The dashed curve represents the numerically calculated eigenvalues of $D_{1}$. The thin curve depicts the variationally calculated eigenvalues with $Z_{1 l}=\operatorname{sech}^{2}(x)-\operatorname{sech}^{2}(x+$ $2 l$ ). The squares signify a better variational calculation of the eigenvalues using $Z_{1 l}=\exp \left(a_{1} x\right)\left[\operatorname{sech}^{2}(x)-\right.$ $\left.\operatorname{sech}^{2}(x+2 l)\right]$. An improved guess of $Z_{2 l}$, and eigenvalue $\lambda_{2 l}$ for the operator $D_{2}$, similarly involves taking $Z_{l 2}=\exp \left(a_{2} x\right)[\operatorname{sech}(x)-\operatorname{sech}(x+2 l)]$. Depicted in Fig. 2(b) are the eigenvalues $\lambda_{2 l}$, numerically calculated (dashed curve), variationally calculated with respective guesses $Z_{2 l}=\operatorname{sech}(x)-\operatorname{sech}(x+2 l)$ (thin line), and $Z_{2 l}=\exp \left(a_{2} x\right)[\operatorname{sech}(x)-\operatorname{sech}(x+2 l)]$ (squares).

The numerical calculation of the eigenvalues $\lambda_{1 l}$ and $\lambda_{2 l}$ involved using a standard QR algorithm on the matrix obtained by a finite difference approximation to the operators $D_{1}$ and $D_{2}$. The grid spacing was adjusted until we obtained convergence. The eigenvectors were calculated using inverse iterations, with the number of iterations optimized for convergence.

The first row in the matrix representation of the adjoint operator Eq. (20) consists only of the operator $D_{1}$. Therefore, since $£^{\dagger} A_{l}^{\dagger}=\lambda_{l} A_{l}^{\dagger}$, we immediately obtain $\lambda_{l}=\lambda_{1 l}$. We recall that in the limit of infinite front distance from the boundary $l \rightarrow \infty$, we have $A_{l}^{\dagger} \rightarrow A^{\dagger}$.
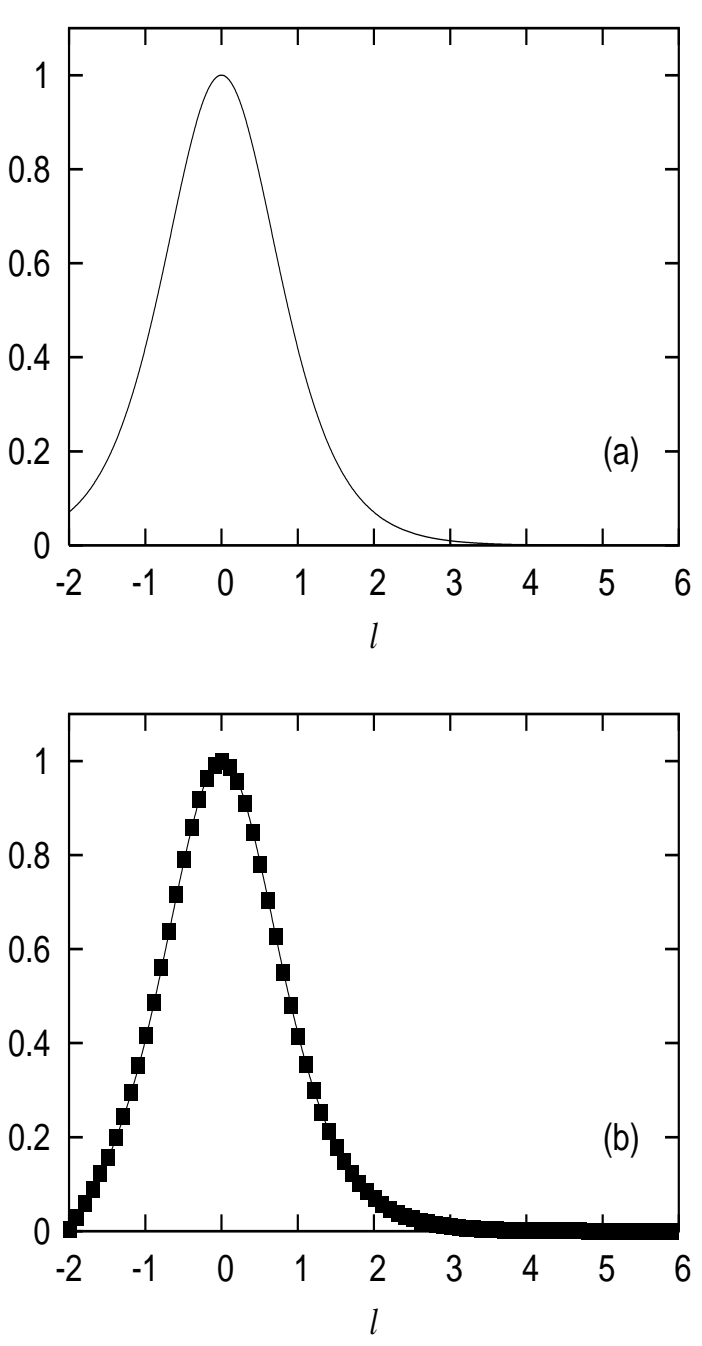

FIG. 1: (a) Shows the plot of $Z_{1}$. The peak is at a distance of $l=2$ from the boundary. (b) The squares represent the numerically obtained $Z_{1 l}$. The analytical guess $Z_{1 l}=\operatorname{sech}^{2}(x)-\operatorname{sech}^{2}(x+2 l)$ is the solid line.

Combining this asymptotic limit constraint with the requirement that the sought after eigenvector has zero values at both boundaries, we obtain,

$$
A_{l}^{\dagger}=\left[\begin{array}{c}
\frac{\left(\mu_{c}-\mu\right) \mu_{c}}{\pi \gamma \nu} Z_{1 l} \\
Z_{2 l}
\end{array}\right] \text {. }
$$

A more rigorous derivation involving a step by step consideration of the operators $L_{1}^{\dagger}$ and $L_{2}^{\dagger}$ in a perturbative scheme also yields Eq. (22).

We now focus on incorporating the effects of the Dirichlet boundary values $X_{b}$ and $Y_{b}$, the values of the real and imaginary components of the field $F$ in Eq. (15), into the dynamics of fronts close to the boundary. Bloch walls are perturbed Ising walls, with the perturbation 

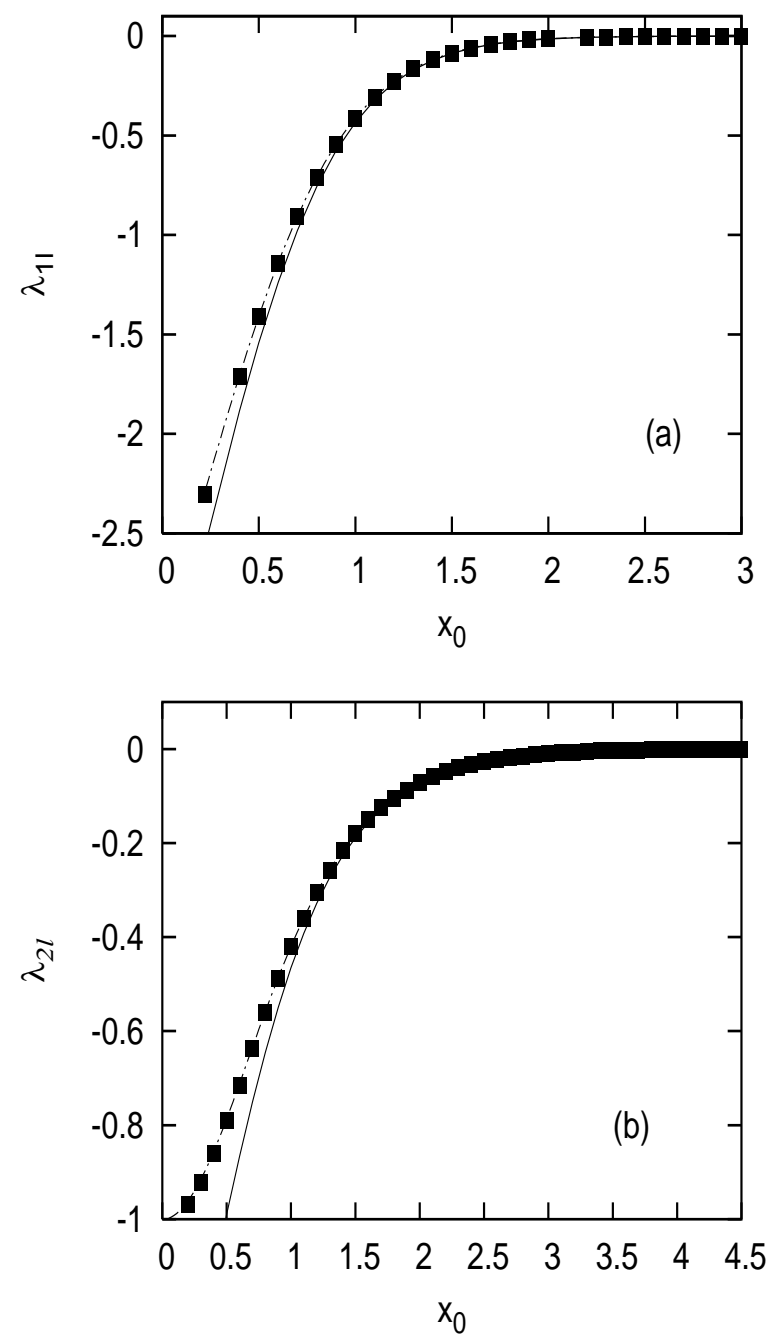

FIG. 2: (a) Comparison of variational and numerical calculations of $\lambda_{1 l}$ (b) Similar comparison of $\lambda_{2 l}$ calculated using numerical and variational techniques.

$\delta U_{l}$. The boundary value of this perturbation $\delta U_{l}(-l)$ is obtained by fixing $F(-l)=X_{b}+i Y_{b}$ and subtracting from it the value that the Ising wall assumes $F_{I}(-l)=$ $\sqrt{\kappa} \tanh (-l) e^{i \phi}$. Recalling Eq. (16), and $\delta U=\{u, w\}^{T}$, we obtain,

$$
\delta U_{l}(-l)=\left[\begin{array}{c}
\left(X_{b} \cos (\phi)+Y_{b} \sin (\phi)\right) / \sqrt{\kappa}+\tanh (l) \\
\left(Y_{b} \cos (\phi)-X_{b} \sin (\phi)\right) / \sqrt{\kappa}
\end{array}\right] .
$$

\section{OPE}

To extract a reduced description of the influence of Dirichlet boundary conditions on the motion of IsingBloch fronts, we invoke Eq. (14), and substitute into it the explicit forms of $A_{l}^{\dagger}$ and $\lambda_{l}$ derived in the previous section. Consider the term $f_{1}=\lambda_{l}\left(c \delta U_{1 l}, A_{l}^{\dagger}\right)$ on the right hand side (RHS) of Eq. (14). For the CGLE, as seen in Eq. (22), the first component of $A_{l}^{\dagger}$, denoted by, $A_{l 1}^{\dagger}$, is smaller by a factor of $c^{2}$ than the second component $A_{l 2}^{\dagger}$. This is so because $\mu_{c}-\mu \sim c^{2}$. Hence, while evaluating $f_{1}$, we need only consider the inner product of the second component of the generalized eigenvector, $\delta U_{1 l}$, denoted by $\delta U_{1 l 2}$, and $A_{l 2}^{\dagger}$. The generalized eigenvector $\delta U_{1}$ is known Eq. (19), and its finite system modification $\delta U_{1 l}$ needs to be evaluated (only the second component $\delta U_{1 l 2}$ ) to evaluate the inner product in $f_{1}$.

To evaluate $\delta U_{1 l 2}$ we recall that $Z_{2}=\operatorname{sech}(x)$, with $D_{2} Z_{2}=0$. The second component of $\delta U_{1}$, is given by $\delta U_{12}=[8 \gamma / 9 \pi \nu] \operatorname{sech}(x)$. Hence, $D_{2} \delta U_{12}=0$. In a confined system with the left boundary at $x=-l, Z_{2}$ is modified to $Z_{2 l}=\operatorname{sech}(x)-\operatorname{sech}(x+2 l)$, requiring that the homogeneous boundary condition, $Z_{2 l}(-l)=0$, holds good. In the confined system $\delta U_{12}$ is modified to $\delta U_{1 l 2}$. However, to obtain $\delta U_{1 l 2}$, the requirement that it obeys the inhomogeneous boundary condition $c \delta U_{1 l 2}(-l)=$ $\delta U_{l 2}(-l)$, since $\delta U_{l}=c \delta U_{1}+\mathcal{O}\left(c^{2}\right)$, needs to be imposed. Therefore we construct $\delta U_{1 l 2}(x)=c \delta U_{12}-\beta \operatorname{sech}(x+2 l)$, followed by imposing the inhomogeneous boundary condition $c \delta U_{1 l 2}(-l)=\delta U_{l 2}(-l)$, to evaluate $\beta$. After doing so, we have,

$$
c \delta U_{1 l 2}=\frac{c 8 \gamma}{9 \pi \nu} Z_{2 l}-\frac{\delta U_{l 2}(-l)}{\operatorname{sech}(l)} \operatorname{sech}(x+2 l) .
$$

We, finally have the ingredients to calculate all the inner products in Eq. (14). The bulk of the boundary influence, we contend, is captured by the interplay of the terms, $c\left(U_{0 x}, A_{l}^{\dagger}\right), \lambda_{l}\left(c \delta U_{1 l}, A_{l}^{\dagger}\right)$, and the surface term $A_{l x}^{\dagger}(-l) \delta U_{l}(-l)$ in Eq. (14). Therefore, although, strictly speaking, the inner products containing higher order terms $c^{2}\left(\delta U_{1 l x}+N_{2}, A_{l}^{\dagger}\right)$, and $c^{3}\left(\delta U_{2 l x}+N_{3}, A_{l}^{\dagger}\right)$, in Eq. (14), should be evaluated in the finite domain $[-l, \infty]$, we approximate them by taking the inner product in the infinite interval $[-\infty, \infty]$.

Performing all the inner products in Eq. (14) and rearranging the terms, we obtain

$$
\begin{aligned}
\partial_{t} c & =\frac{27\left(\mu_{c}-\mu\right) \mu_{c}}{4 \gamma^{2}} c+\lambda_{l} c-\left(\left[\frac{8 \gamma}{9 \pi \nu}\right]^{2}+p\right) c^{3} \\
& -\left[\frac{9 \pi \nu}{16 \gamma}\right] \tanh (l) \operatorname{sech}(l) \delta U_{l 2}(-l) \\
& +\left[\frac{81\left(\mu_{c}-\mu\right) \mu_{c}}{4 \gamma^{2}}\right] \tanh (l) \delta U_{l 1}(-l) \\
& -\left[\lambda_{l} \frac{9 \pi \nu}{16 \gamma}\right] 2 l \operatorname{cosech}(2 l) .
\end{aligned}
$$

In deriving Eq. (25) we have used $Z_{1 l}=\operatorname{sech}^{2}(x)-$ $\operatorname{sech}^{2}(x+2 l)$ and $Z_{2 l}=\operatorname{sech}(x)-\operatorname{sech}(x+2 l)$, where $\lambda_{l}=\lambda_{1 l}$ is given by Eq. (21), and $p=0.36$ Eq. (19). Equation. 25. along with $\partial_{t} l=-c$ represents the coupling of the two degrees of freedom, front velocity $c$ and 
position $l$, by the influence of Dirichlet boundary conditions imposed at the boundary. As required, in the limit of infinite front distance from the boundary Eq. (25) reduces to Eq. (19).

We now examine the consequences of the coupling of the front velocity and position close to the boundary. Firstly, we report the findings of our numerical simulations of Eq. (15), which is a system with infinite degrees of freedom. Secondly, we corroborate these findings by solving the reduced, two degree of freedom OPE we have derived.

We performed numerical simulations of Eq. (15), where Bloch fronts were created at infinity (far from the boundaries) and launched towards a boundary. The velocity of these Bloch fronts was chosen to be one of the steady states of Eq. (19) resulting in uniform front translation with this velocity until the fronts closed in on the boundary. Near the boundary, contingent upon the Dirichlet boundary value imposed, the incoming Bloch fronts were either trapped or bounced back. Bloch fronts that bounce evolve into the counter-propagating Bloch front near the boundary and move away. Trapped Bloch fronts, as opposed to bouncing Bloch fronts, evolve into nontrivial steady state solutions (See Ref. 12]) of the CGLE Eq. (15).

We summarize our numerical observations of Bloch front behavior as a function of the boundary conditions $X_{b}$ and $Y_{b}$ in Figure. 3. This phase diagram in the plane of boundary values reveals a curve separating regions of bouncing and trapped fronts represented by diamonds. We compare these results with the transition curve predicted by the reduced model Eq. (25), plotted as the dashed curve in Figure. 3] The plots show a good agreement (within $0.5 \%$ ) between the two transition curves. This is a striking result considering the fact that in calculating $A_{l}^{\dagger}$ and $\lambda_{l}$ we have employed approximate vectors $Z_{1 l}$ and $Z_{2 l}$.

Bouncing fronts gradually slow down as they near the boundary, attain zero velocity at a certain critical distance from it, and finally move away as the sign of the velocity flips. As we change the boundary values and get closer to the transition curve, bouncing fronts attain zero velocity at a much smaller critical distance from the boundary, until eventually right at the transition curve they reach the point of closest approach to the boundary. As we cross the transition curve and move into the trapping region, approaching fronts no longer attain zero velocity close to the boundary, their velocity never flips sign, and hence they never bounce. The distance from the boundary of the point of closest approach depends on where exactly on the phase diagram the transition curve is crossed.

The agreement between the transition curves obtained from the full model Eq. (15) and the reduced model Eq. (25) is better when the point of closest approach is further away from the boundary. This is because, as detailed earlier, the vectors $Z_{1 l}$ and $Z_{2 l}$ are better approximations to the actual solutions of $D_{1} Z_{1 l}=\lambda_{1} Z_{1 l}$ and

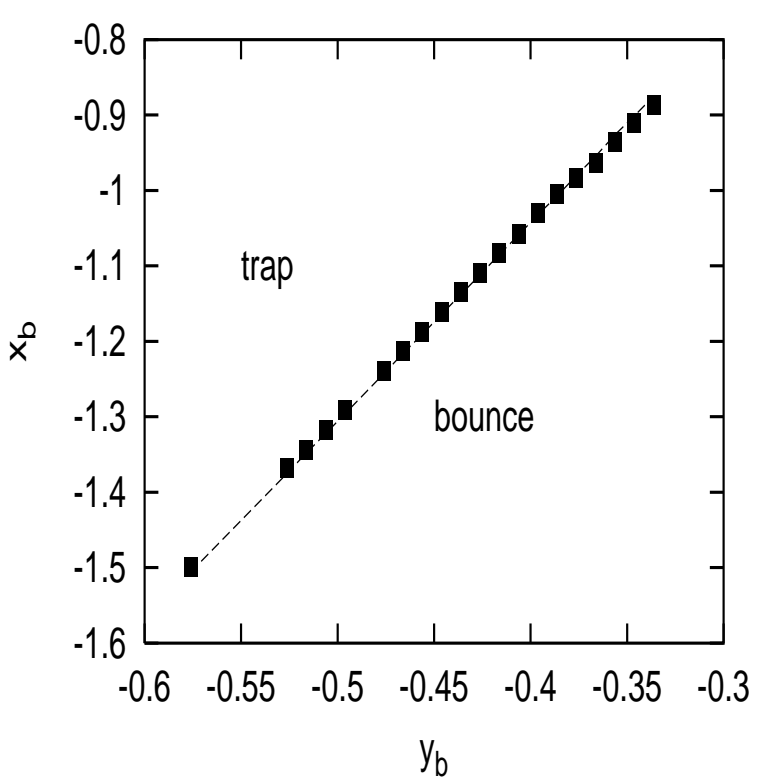

FIG. 3: The transition curve for the full model Eq. (15) plotted using squares, the same curve obtained from the reduced OPE Eq. (25), plotted as a dashed line. Here, $\nu=0.3$, $\gamma=1.0, \mu=0.448$.

$D_{2} Z_{2 l}=\lambda_{2} Z_{2 l}$, further away from the boundary. Consequently, a better guess of these vectors, valid close to the boundary, should improve the agreement between the transition curves, even if, the point of closest approach is closer to the boundary. However, the approximate vectors we use are sufficient for the purpose of establishing the usefulness of our general method that accounts for the broken translational invariance in a spatially finite system through the extension of solvability conditions. Our method incorporates into it the eigenvalue $\lambda_{l}$, the most direct measure of broken translational invariance, which can be obtained accurately via a variational principle using relatively crude guesses for the eigenvectors.

We now, by examining Eq. (25) in more detail, extract the mechanism behind the transition from bouncing to trapped fronts as Dirichlet boundary conditions are changed. Figure. 四(a) shows the nullclines, invariant manifold, and trajectories of Eq. (25) inside the bouncing region of the phase diagram. A saddle, present at the point of intersection of the nullclines, controls the flows in this bouncing regime. Far away from the boundary, situated at $x=0$ in the plot, the nullclines are three parallel straight lines that represent two counter-propagating Bloch wall steady state solutions, and a stationary Ising wall solution of Eq. (19). The bouncing involves the Bloch front initially flowing towards the saddle. Thereupon, influenced by the unstable manifold, the front flows away.

Figure. 4(b) still depicts flows inside the bouncing region, but much closer to the transition curve. In this regime bouncing and trapped fronts can coexist. The invariant manifolds demarcate two basins, one of attraction 

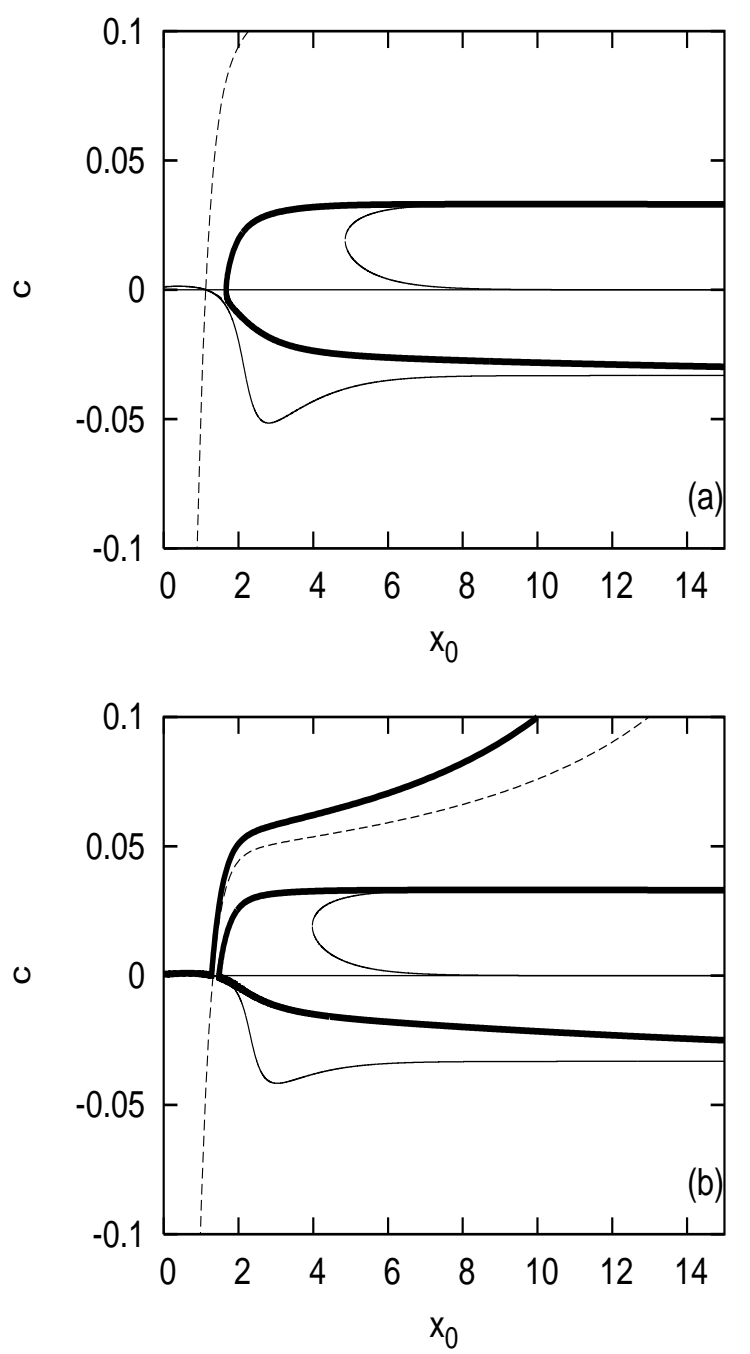

FIG. 4: (a) The plot deep inside the bouncing region, the nullclines are thin black curves, the thick curves correspond to the trajectories in the phase plane, and the invariant manifolds are plotted as dashed lines. Here, $\nu=0.3, \gamma=1.0$, $\mu=0.448, X_{b}=-1.116$, and $Y_{b}=-0.4262$. (b) Plot still in the bouncing region, but close to the transition curve. The same plotting scheme and parameters used, with boundary values $X_{b}=-1.112, Y_{b}=-0.4262$.

towards the boundary, and the other of repulsion away from it. Inside the repulsion basin all incoming Bloch fronts bounce with the same mechanism as in Fig. 目(a). All the flows in the attraction basin are directed towards the system boundary, with no possibility of a bounce. Figure. [(b) shows both bouncing and trapped Bloch front trajectories in their respective basins. We reported on the the coexistence region in our numerical study of Eq. (15) in Ref. 12]. Here, we have provided an analytical explanation of this phenomena.

The flows in the trapping region close to the transition curve are shown in Figure. 5(a). Trapped Bloch fronts, created at infinity and on the upper branch of the
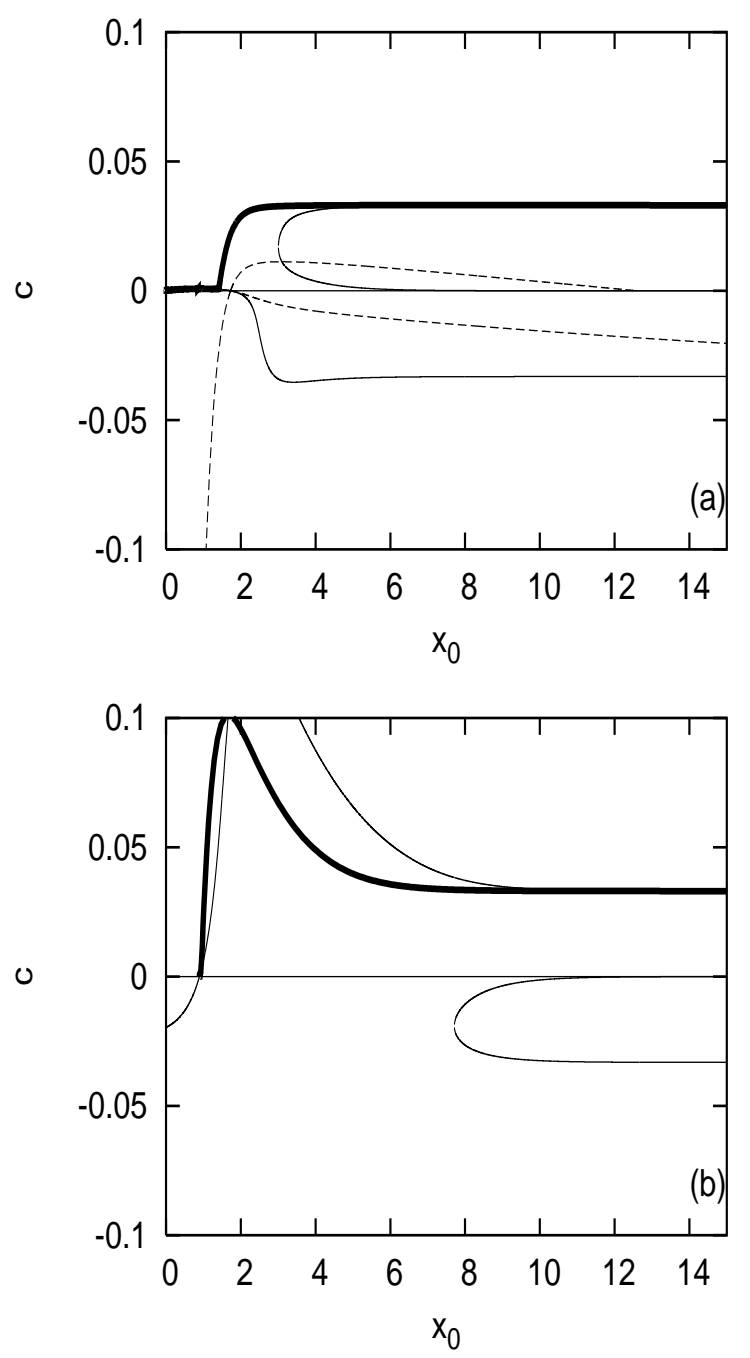

FIG. 5: (a) Plot in the trapping region close to the transition curve. The same plotting scheme and parameters used, with boundary values $X_{b}=-1.11, Y_{b}=-0.4262$. (b) The plot deep inside the trapping region, the nullclines are thin black curves, the trajectory is the thick curve. Here, $\nu=0.3, \gamma=$ 1.0, $\mu=0.448, X_{b}=-1.09$, and $Y_{b}=-0.4262$.

nullcline (corresponding to one of the steady states of Eq. (19), lie inside the basin of attraction towards the boundary. Consequently, the transition from bouncing to trapped fronts is marked by the initial front velocity and position moving from the basin of repulsion (Fig. [4(b)) to the basin of attraction (Figure. 5(a)) as the boundary values are varied. Deep inside the trapping region the saddle no longer exists, and we have a sink instead (Fig. 罒(b)). All incoming Bloch front trajectories end up at this sink.

Summarizing, the nonuniform motion of Bloch fronts close to the boundary is governed by the fixed point of Eq. (25), giving rise to bouncing, trapping, and coexistence of the two. Well inside the bouncing region this fixed point is a saddle. Deep into the trapping region the 
fixed point changes into a sink.

\section{CONCLUSION}

We have developed a general method of analyzing the influence of broken translational invariance due to finite size and boundary effects on the dynamics of localized solutions of generic non-linear spatially extended systems. We apply our method to the special case of a bistable reaction-diffusion system, where the localized solutions are fronts Eq. (25). The implementation of this method involves the extension of the infinite system size limit solvability conditions, used to extract a reduced description of the infinite dimensional system, into solvability conditions that account for finite system size and boundary effects. The extended solvability criteria works by naturally incorporating into it the concept of images. As a result, the method affords a direct grasp of the broken translational invariance in a confined system through the calculation of relevant eigenvalues.

In the special case of Dirichlet boundary conditions imposed on the CGLE, we were able to provide mechanisms for Bloch front trapping, bouncing and coexistence of the two at the boundary. This nonuniform front motion is a result of the coupling of the two degrees of freedom, front velocity and position, by the influence of boundary conditions. We have explicitly derived this coupling by using our method of solvability condition extension. The role of other types of boundary conditions, either Neumann or mixed can be explored in a similar fashion by constructing a suitable extension of the modified Goldstone mode. For example, exploring Neumann boundary conditions requires the extension to always have zero derivatives at the boundary. This can be accomplished in the CGLE or other systems by adding, rather than subtracting, the image.

Finally, we comment on the generality of solvability condition extension. In any system, whenever it is possible to derive reduced dynamical equations through pro- jections on the Goldstone mode, our method can be applied to obtain the finite size and boundary effects in terms of the modifications of these reduced dynamical equations.

\section{Acknowledgments}

This work was supported in part by NSF Grant No. DMR-9710608 and by a Faculty Research Grant from the Louisiana State University office of Sponsered Research.

\section{APPENDIX A}

For the CGLE, consider the operator $D_{1}$ Eq. (17) in a semi-infinite interval $[-l, \infty]$. Using the transformation $t=1-e^{-(l+x)}$, the problem

$$
\begin{aligned}
D_{1} Y & =\left[\partial_{x}^{2}+2-6 \tanh ^{2}(x)\right] Y=0 \\
Y(-l) & =0 ; Y(\infty)=0
\end{aligned}
$$

is transformed to

$$
\begin{aligned}
& {\left[\partial_{t}^{2}-\frac{\partial_{t}}{(1-t)}+\frac{2+6 \tanh ^{2}(l+\ln (1-t))}{(1-t)^{2}}\right] Y=0} \\
& Y(0)=0 ; Y(1)=0 .
\end{aligned}
$$

Equation. A2 has a regular singular point at $t=1$, and thus has a unique solution. Similar considerations apply to the operator $D_{2}$. Therefore, homogeneous or inhomogeneous problems involving the operator $£$, which is comprised of the operators $D_{1}$, and $D_{2}$, should have unique solutions in a semi-infinite domain. For operators that possess exponential decay asymptotics (true for a wide variety of models of physically occurring localized structures), a transformation of the type used here, can always be found in order to prove the uniqueness.
[1] M. C. Cross and P. C. Hohenberg, Rev. Mod. Phys. 65, 851 (1993).

[2] C. Elphick, E. Meron, E. A. Spiegel, SIAM. J. Appl. Math. 50, 490 (1990).

[3] C. Elphick, E. Meron, Phys. Lett. A. 230, 33 (1997).

[4] A. Hagberg, E. Meron, I. Rubinstein, and B. Zaltzman, Phys. Rev. Lett. 76, 427 (1996).

[5] D. V. Skryabin, A. Yulin, D. Michaelis, W. J. Firth, G. L. Oppo, U. Peschel, and F. Lederer, Phys. Rev. E. 64, 056618 (2001).

[6] P. C. Fife, SIAM. J. Appl. Math. 48, 506 (1988)

[7] E. Meron, Phys. Rep. 218, 1 (1992).

[8] H. Ikeda, M. Mimura, and Y. Nishiura, Nonl. Anal. TMA. 13, 507 (1989).

[9] A. Hagberg and E. Meron, Phys. Rev. Lett. 72, 2494 (1994).
[10] A. Hagberg and E. Meron, Chaos 4, 477 (1994).

[11] D. Haim, G. Li, Q. Ouyang, W. D. McCormick, H. L. Swinney, A. Hagberg, and E. Meron, Phys. Rev. Lett. 77, 190 (1996).

[12] A. Yadav, D. A. Browne, Phys. Rev. E. 70, 036218 (2004).

[13] U. Ebert and W. van Saarloos, Phys. Rep. 337139 (2000).

[14] P. Coullet, J. Lega, B. Houchmanzadeh, and J. Lajzerowicz, Phys. Rev. Lett. 65, 1352 (1990).

[15] G. Haas, M. Bar, I. G. Kevrekidis, P. B. Rasmussen, H.H. Rotermund, and G. Ertl, Phys. Rev. Lett. 75, 3560 (1995).

[16] G. Li, Q. Ouyang, and H. L. Swinney, J. Chem. Phys. 105, 10830 (1996).

[17] T. Frisch, S. Rica, P. Coullet, and J. M. Gilli, Phys. Rev. 
Lett. 72, 1471 (1994).

[18] S. Nasuno, N. Yoshimo, and S. Kai, Phys. Rev. E. 51, 1598 (1995).

[19] Liquid crystals in complex geometries: formed by polymer and porous networks, Edited by Phillip Crawford and Slobodan Zumer, Taylor and Francis (1996).
[20] M. Bode, A. Reuter, R. Schmeling and H. -G. Purwins, Phys. Lett. A. 185, 70 (1994).

[21] D. Michaelis, U. Peschel, F. Lederer, D. V. Skryabin, and W. J. Firth, Phys. Rev. E. 63, 066602 (2001).

[22] M. Bode, Physica. D. 106, 270 (1997). 